Cahiers
de a Recherche
sur les Droits

Cahiers de la recherche sur les droits fondamentaux

16 | 2018

Les partis politiques

\title{
L'histoire dans le contentieux des partis politiques devant la Cour européenne des droits de l'homme
}

History in Party-Political Litigation before the European Court of Human Rights

Yannick Lécuyer

\section{(2) OpenEdition}

Édition électronique

URL : https://journals.openedition.org/crdf/323

DOI : $10.4000 /$ crdf.323

ISSN : 2264-1246

Éditeur

Presses universitaires de Caen

Édition imprimée

Date de publication : 16 novembre 2018

Pagination : 101-110

ISBN : 978-2-84133-901-3

ISSN : $1634-8842$

Référence électronique

Yannick Lécuyer, "L'histoire dans le contentieux des partis politiques devant la Cour européenne des droits de l'homme », Cahiers de la recherche sur les droits fondamentaux [En ligne], 16 | 2018, mis en ligne le 16 novembre 2019, consulté le 14 novembre 2022. URL : http://journals.openedition.org/crdf/ 323 ; DOI : https://doi.org/10.4000/crdf.323

Tous droits réservés 


\title{
L'histoire dans le contentieux des partis politiques devant la Cour européenne des droits de l'homme
}

\author{
Yannick LÉCUYER \\ Maître de conférences en droit public (HDR) à l'université d'Angers \\ Collaborateur de la Fondation René Cassin
}

I. L'histoire, outil de modulation du contrôle européen

A. La dilatation de la marge d'appréciation en matière de libertés des partis politiques

B. La fragmentation des standards démocratiques

II. Une vision dynamique et volontariste de l'histoire

A. Le caractère facultatif de l'histoire des États dans le contentieux des partis politiques

B. La fabrique de l'histoire politique et démocratique européenne

Elle-même née dans un contexte historique particulier, sur les cendres du conflit le plus meurtrier que l'humanité ait jamais engendré et à l'aube d'une nouvelle guerre, froide celle-ci, la Cour européenne des droits l'homme n'est pas indifférente à l'histoire ${ }^{1}$. Loin s'en faut. L'histoire est fréquemment discutée dans la jurisprudence du juge de Strasbourg. Toutefois, si elle a fait quelques apparitions spectaculaires à propos des ingérences relatives aux débats historiques sur les épisodes tragiques et génocidaires du
$\mathrm{XX}^{\mathrm{e}}$ siècle ${ }^{2}$, c'est surtout en tant qu'outil du contrôle juridictionnel que sa présence est la plus remarquable. Ainsi que l'observait Jean-François Flauss, la Cour connaît de contentieux

[...] étroitement liés à d'importants événements historiques tels entre autres ceux consécutifs à la Seconde Guerre mondiale, à des changements révolutionnaires de régimes politiques, à des occupations ou des actions militaires ${ }^{3}$.

1. Voir La loi peut-elle dire l'histoire? Droit, justice et histoire, B. Favreau (dir.), Bruxelles, Bruylant, 2012 (en particulier: C. Pettiti, «La Cour européenne des droits de l'homme et l'histoire de la France», p. 41-62; M. Puéchavy, «L'histoire de la Seconde Guerre mondiale dans la jurisprudence de la Cour européenne des droits de l'homme», p. 63-88; et J.-F. Flauss, "L'histoire dans la jurisprudence de la Cour européenne des droits de l'homme: le contentieux des anciennes démocraties populaires», p. 89-101); A. Kovler, "La Cour devant l'histoire, l'histoire devant la Cour", in La conscience des droits. Mélanges en l'honneur de Jean-Paul Costa, P. Titiun, P. Dumaine (dir.), Paris, Dalloz, 2011, p. 337-352; J.-M. Sauvé, "L'histoire, le droit et les juges", in La conscience des droits..., p. 587-596.

2. Cour EDH, 24 juin 2003, Garaudy c. France (déc.), nº 65831/o1; Cour EDH, 7 juin 2011, Gollnisch c. France (déc.), nº 48135/o8; Cour EDH, 17 décembre 2013, Perinçek $c$. Suisse, $\mathrm{n}^{\circ}$ 27510/o8. La Cour considère que la recherche des vérités historiques relève de la liberté d'expression et qu'il ne lui appartient pas d'arbitrer les débats entre historiens. Toutefois, elle invite les États à débattre ouvertement et sereinement de leur propre histoire (Cour EDH, 21 septembre 2006, Monnat c. Suisse, $\mathrm{n}^{\circ}$ 73604/01, \$64). Voir également J. Morange, "Histoire et liberté d'expression", Les cahiers de droit, vol. $53, \mathrm{n}^{\circ} 4,2012$, p. $715-737$.

3. J.-F. Flauss, «L'histoire dans la jurisprudence de la Cour européenne des droits de l'homme», Revue trimestrielle des droits de l'homme, ${ }^{\circ}$ 65, 2006, p. 5-22, spéc. p. 6. 
L'histoire joue un rôle non négligeable, en général au profit d'un élargissement de la marge nationale d'appréciation et, partant, afin de réduire l'intensité du contrôle européen. Les exemples sont légion : décision d'irrecevabilité Birk-Levy c. France du 21 septembre 2010 qui survalorise les considérations d'ordre historique et politique à l'origine des singularités institutionnelles et constitutionnelles françaises, arrêts Folgero et autres c. Norvège du 29 juin 2007, Zengin c. Turquie du 9 octobre 2007 ou Lautsi c. Italie du 18 mars 2011 qui tolèrent une asymétrie au profit des religions qui occupent une place particulière dans l'histoire et la tradition des États défendeurs ${ }^{4}$. Dans l'arrêt Lautsi c. Italie, la grande chambre insiste sur la liberté des États de perpétuer ou non leurs traditions, laquelle relève de leur marge d'appréciation ${ }^{5}$. Encore plus significatif, l'arrêt Rohlena c. République tchèque du 27 janvier 2015 replace la notion d'infraction pénale dans son contexte historique et son régime dans les quarante-sept États membres du Conseil de l'Europe avant de conclure à l'absence de violation de l'article 7 de la Convention ${ }^{6}$. Ce sont les pays d'Europe centrale et orientale anciennement sous le joug soviétique qui sont les plus grands pourvoyeurs d'affaires nécessitant un éclairage historique. Ainsi, à l'instar de bien d'autres, l'arrêt Kononov c. Lettonie du 17 mai 2010 relatif à la conventionnalité de la condamnation pour crime contre l'humanité du requérant, ancien membre d'un commando soviétique composé de "partisans rouges» durant l'occupation allemande, comporte une analyse historiographique aussi riche que détaillée. La Cour étudie minutieusement les faits historiques à prendre en considération ainsi que le droit applicable au moment des faits 7 . L'histoire des plus anciens membres du Conseil de l'Europe n'est pas négligée. On retrouve une démarche analogue dans la décision Gecel c. France du 15 novembre 2011 ou l'arrêt Jahn et autres c. Allemagne du 22 janvier 2004 relatif au droit de propriété (art. $1^{\text {er }}$ du Protocole $n^{\circ} 1$ ), la déportation servant de toile de fond dans le premier cas et la réunification allemande, " contexte historique unique», dans le second ${ }^{8} .$.

C'est indubitablement dans le cadre du contentieux des droits politiques, droit à des élections libres, et des droits qui en permettent l'animation démocratique, droits de nature politique que l'histoire apparaît le plus souvent, peut-être parce que les droits politiques touchent à l'identité constitutionnelle des États. Par conséquent, leur mise en œuvre «ne saurait alors être comprise sans l'appréhension d'un certain contexte historique et politique national ${ }^{9}$. De fait, les ingérences dans la liberté des partis politiques sont rarement dissociables du contexte historique et politique précis même si, en apparence, le problème de conventionnalité semble purement technique. En 2016, le rejet pour défaut manifestement de fondement et non-épuisement des voies de recours internes de la requête présentée par le NPD, parti d'extrême droite allemand, s'inscrit dans un contexte d'endiguement par les autorités allemandes des résurgences nationales-socialistes ${ }^{10}$. Comment appréhender les multiples affaires de dissolution des partis politiques prokurdes en Turquie - PCU, Parti socialiste, ÖZDEP, HEP, DBP, HADEP, DTP... - sans s'intéresser préalablement au conflit qui agite le Kurdistan depuis 1984, conflit qui s'enracine lui-même dans la création autoritaire de l'État turc sur les ruines de l'Empire ottoman ${ }^{11}$ ? C'est vers le tropisme politique et historique turc pour l'autoritarisme qu'il faut regarder si l'on souhaite comprendre l'arrêt Cumhuriyet Halk Partisi (CHP) c. Turquie du 26 juillet $2016^{12}$. De prime abord, l'affaire concerne uniquement la confiscation des actifs du parti politique à la suite d'un contrôle de ses comptes. Toutefois, ce parti était aussi un des principaux membres de l'opposition. L'affaire s'inscrit dans une dynamique d'affaiblissement du pluralisme en Turquie et de glissement de la démocratie vers la démocrature ${ }^{13}$. C'est également un problème de financement et de transparence financière, en l'occurrence des subventions venant d'un pays étranger, qui était en cause dans l'affaire Parti nationaliste basque c. France du 7 juin 2007. Là encore, le contexte historique est essentiel même s'il n'est pas discuté. L'arrêt doit être lu en parallèle avec l'arrêt Herri Batasuna et Batasuna c. Espagne du 30 juin 2009 qui interroge les velléités séparatistes basques de l'autre côté des Pyrénées. En toutes hypothèses, l'histoire et les tensions qu'elle charrie sont toujours présentes.

Sans surprise, les États défendeurs tentent régulièrement d'utiliser leur histoire nationale à des fins absolutoires. Le moyen est à la fois souple, malléable et pratique. L'histoire peut facilement être manipulée, réinterprétée,

4. Cour EDH, 29 juin 2007, Folgero et autres c. Norvège, $n^{\circ}$ 15472/02, \$ 89; Cour EDH, 9 octobre 2007, Zengin c. Turquie, 46928/99, $\$ 63 ;$ Cour EDH, 18 mars 2011, Lautsi c. Italie, $\mathrm{n}^{\circ} 30814 / 06, \$ 71$

5. Cour EDH, 18 mars 2011, Lautsi c. Italie, $\$ 68$

6. Cour EDH, 27 janvier 2015, Rohlena c. République tchèque, $\mathrm{n}^{\circ}$ 59552/o8, $\$ 30$ sq.

7. Cour EDH, 17 mai 2010, Kononov c. Lettonie, $\mathrm{n}^{\circ} 36376 / 04, \$ 188$ sq.

8. Cour EDH, 15 novembre 2011, Gecel c. France (déc.), n 63628/o9; Cour EDH, 22 janvier 2004, Jahn et autres c. Allemagne, $\mathrm{n}^{\circ}$ 46720/99, 72203/o1, $72552 / 01, \S 112$.

9. X. Souvignet, «Le modèle politique de la Cour européenne des droits de l'homme: du pouvoir du peuple à la souveraineté du sujet », Jurisdoctoria, $\mathrm{n}^{\circ} 5,2010$, p. 43 .

10. Cour EDH, 4 octobre 2016, Nationaldemokratische Partei Deutschlands (NPD) c. Allemagne, $\mathrm{n}^{\circ} 55977 / 13$.

11. Cour EDH, 30 janvier 1998, Parti communiste unifié de Turquie (PCU) et autres c. Turquie, $\mathrm{n}^{\circ}$ 19392/92; Cour EDH, 25 mai 1998, Parti socialiste et autres c. Turquie, $\mathrm{n}^{\circ}$ 21237/93; Cour EDH, 8 décembre 1999, Parti de la liberté et de la démocratie (ÖZDEP) c. Turquie, $\mathrm{n}^{\circ} 23885 / 94 ; \mathrm{Cour} \mathrm{EDH}$, 9 avril 2002, Yazar, Karataş, Aksoy et le Parti du travail du peuple (HEP) c. Turquie, nº 22723/93, 22724/93, 22725/93; Cour EDH, 12 juillet 2005, Güneri c. Turquie, no 42853/98, 43609/98, 44291/98; Cour EDH, 14 décembre 2010, HADEP et Demir c. Turquie, nº 28oo3/o3; Cour EDH, 12 janvier 2016, Parti pour une société démocratique (DTP) et autres c. Turquie, nº 3840/10, 3870/10, 3878/10, 15616/10, 21919/10, 37272/10, 39118/10.

12. Cour EDH, 26 juillet 2016, Cumhuriyet Halk Partisi (CHP) c. Turquie, nº 19920/13.

13. Mutadis mutandis, la même remarque vaut pour l'arrêt Parti républicain de Russie c. Russie du 12 avril 2011 ( $\left.\mathrm{n}^{\circ} 12976 / 07\right)$, parti dissous pour non-respect des prescriptions légales relatives au nombre minimal d'adhérents. 
voire réécrite pour servir des intérêts politiques ou sociaux, ici s'exonérer de sa responsabilité devant une juridiction internationale. Le piège tendu au juge européen est redoutable. Il l'oblige à s'improviser historien le temps d'une audience, ce qui lui sera ensuite sévèrement reproché. L'ancien juge Giovanni Bonello (1998-2010) en avait même fait le fond de commerce de ses dissidences acrimonieuses. Fervent partisan de la marge d'appréciation en matière de droits politiques, il reprochait notamment à l'arrêt Ždanoka c. Lettonie du 17 juin 2004 d'avoir substitué un

[...] credo politico-historique classique à celui d'un État qui a perdu la démocratie grâce aux exploits de personnes comme la requérante, l'a reconquise malgré les luttes impénitentes de personnes dans son genre et la conserve en dépit de l'ardent désir de personnes semblables à elle ${ }^{14}$.

Le contentieux relatif aux partis politiques est particulièrement sensible. Il l'est matériellement puisque, depuis la reconnaissance de l'applicabilité de l'article 11 de la Convention et de la liberté d'association, la Cour ne cesse de souligner le rôle primordial qu'ils occupent dans un régime démocratique. Les partis politiques participent $\mathrm{au}$ «libre jeu du débat politique» qui se trouve au «cœur même de la notion de société démocratique ${ }^{15}$. Il l'est ensuite parce que leur liberté mobilise plusieurs droits conventionnels vitaux en démocratie: la liberté d'association et la liberté de réunion pacifique (art. 11), la liberté d'expression (art. 10) et le droit à des élections libres (art. 3 du Protocole $n^{\circ} 1$ ), lequel ne saurait se concevoir «sans le concours d'une pluralité de partis politiques représentant les courants d'opinions qui traversent la politique d'un pays ${ }^{16}$. Ils apportent une "contribution irremplaçable au débat politique $»^{17}$ en répercutant ces courants dans les institutions politiques, dans les médias et à tous les niveaux de la vie en société. Cette lecture triangulaire des articles 10, 11 de la Convention et 3 du Protocole $n^{\circ} 1$ les situe au centre de gravité de la vie politique des États et les érige en «avant-garde de la liberté d'opinion politique ${ }^{18}$. Il s'agit de donner vie au pluralisme afin d'assurer une représentation démocratique. Enfin, ce contentieux est délicat car, plus qu'aucun autre, il mobilise la compréhension d'un environnement historique et sociétal parfois très difficile.

Subséquemment, la Cour a pris en compte de nombreuses périodes et événements historiques afin d'apprécier la pertinence et la proportionnalité des mesure étatiques: «cauchemar du nazisme» et "amère période» suivant l'effondrement de la république de Weimar ${ }^{19}$, processus de réunification de la République fédérale et de la République démocratique allemandes ${ }^{20}$, appartenance de la République populaire de Hongrie au bloc soviétique entre 1949 et $1989^{21}$, théocratie ottomane jusqu'à la disparition de l'empire en $1922^{22}$, fondement historique du système de la fonction publique ${ }^{23}$... Dans l'arrêt Ždanoka c. Lettonie du 16 mars 2006, la grande chambre consacre de longs développements à la genèse de l'affaire et le contexte historique. Plusieurs paragraphes balayent l'histoire du pays depuis le début de la période soviétique jusqu'à son adhésion à l'Union européenne en $2004^{24}$. Dans l'arrêt Gorzelik c. Pologne du 17 février 2004, c'est un véritable cours d'histoire médiévale qui est dispensé avec l'évocation de l'occupation de la Silésie par le royaume de Bohème au $\mathrm{XIV}^{\mathrm{e}}$ siècle puis par l'Autriche au $\mathrm{XVI}^{\mathrm{e}}$ siècle $^{25}$.

Nonobstant, si l'histoire s'est imposée comme un outil habituel de modulation du contrôle des ingérences dans la liberté des partis politiques (I), avec toutes les difficultés que cela comporte, la démarche de la Cour est moins celle d'un historien que celle d'un véritable acteur de l'histoire (II).

\section{L'histoire, outil de modulation du contrôle européen}

Plus que dans toute autre partie du contentieux européen des droits de l'homme, la contextualisation historique s'est banalisée dans le contentieux relatif aux partis politiques. Elle s'y traduit par une dilatation de la marge d'appréciation accordée aux États (A) et un dédoublement regrettable des standards démocratiques $(\mathrm{B})$.

\section{A. La dilatation de la marge d'appréciation en matière de libertés des partis politiques}

A priori, les exceptions visées à l'article $11, \$ 2$ de la Convention appellent une interprétation des plus strictes à l'égard des mesures qui restreignent la liberté des partis politiques: «[...] seules des raisons convaincantes et impératives pouvant justifier des restrictions à leur liberté d'association. Pour juger en pareil cas de l'existence d'une nécessité au sens de l'article $11 \$ 2$, les États contractants ne disposent que d'une marge d'appréciation réduite», doublée «d'un

14. Cour EDH, 17 juin 2004, Ždanoka c. Lettonie, $\mathrm{n}^{\circ}$ 58278/oo, opinion dissidente du juge Bonello, $\$ 3.5$.

15. Cour EDH, 8 juillet 1986, Lingens $c$. Autriche, no 9815/82, $\$ 42$.

16. Cour EDH, 30 janvier 1998, Parti communiste unifié de Turquie..., $\$ 44$.

17. Ibid.

18. J.-F. Flauss, «Le droit constitutionnel devant les instances de contrôle de la Convention européenne des droits de l'homme», Revue française de droit constitutionnel, $\mathrm{n}^{\circ} 30,1997$, p. 399.

19. Cour EDH, 26 septembre 1995, Vogt c. Allemagne, $\mathrm{n}^{\circ}$ 17851/91, $\$ 51$ et 59 .

20. Cour EDH, 12 décembre 2002, Wittek c. Allemagne, $\mathrm{n}^{\circ} 37290 / 97, \$ 15,25$ sq.

21. Cour EDH, 20 mai 1999, Rekvényi c. Hongrie, $\mathrm{n}^{\circ}$ 25390/94, $\$ 47$.

22. Cour EDH, 13 février 2003 , Refah Partisi (Parti de la prospérité) et autres c. Turquie, $\mathrm{n}^{\circ}$ 41340/98, 41342/98, 41343/98, 41344/98, $\$ 125$

23. Cour EDH, 2 septembre 1998, Ahmed c. Royaume-Uni, n² 22954/93, \$62.

24. Cour EDH, 16 mars 2006, Ždanoka c. Lettonie, $\$ 12$ sq.

25. Cour EDH, 17 février 2004, Gorzelik c. Pologne, n $44158 / 98, \S 13$. 
contrôle européen rigoureux portant à la fois sur la loi et sur les décisions qui l'appliquent, y compris celles d'une juridiction indépendante ${ }^{26}$. Cette affirmation est présente dès l'arrêt fondateur Parti communiste unifié c. Turquie. Elle est a été répétée avec force dans l'arrêt Refah Partisi c. Turquie du 13 février 2003 et ne sera plus jamais remise en cause sur le principe ${ }^{27}$. Des mesures aussi sévères que la dissolution du parti politique ou l'interdiction pour ses responsables d'exercer une activité politique doivent être réservées aux cas les plus graves ${ }^{28}$.

Pourtant, la Cour atténue parfois la portée de ce credo itératif grâce à une contextualisation historique. Comme l'observe Laurence Burgorgue-Larsen, le processus repose toujours sur les mêmes présupposés: «[...] ne pas ignorer, au risque de les bafouer, des éléments inhérents à l'histoire, la sociologie politique, le droit des États membres ${ }^{29}$. Les considérations d'ordre historique sont devenues un paramètre de détermination de l'ampleur de la marge d'appréciation concédée aux États, plus précisément de son élargissement ${ }^{30}$. Elles ne renvoient à aucun standard européen commun et laissent aux autorités une latitude considérable ${ }^{31}$.

La méthode conduit à préférer une interprétation de la Convention «à la lumière de l'évolution politique du pays» ou «à la lumière de l'histoire de ce pays». Elle neutralise l'interprétation dynamique et évolutive ordinairement utilisée à Strasbourg, celle qui se fait à «la lumière des conditions de vie actuelle» ou fondée «sur les conceptions prévalant de nos jours dans les sociétés démocratiques $»^{32}$. Partant, son examen global tient compte de l'évolution historique dans laquelle se situe la dissolution du parti politique, par exemple l'importance de l'intérêt général à préserver le principe de laïcité en Turquie pendant l'évolution de ce pays afin de favoriser «le bon fonctionnement de la société démocratique ${ }^{33}$. Le procédé est en réalité contre-productif. Non seulement, il s'agit d'un frein à la modernisation de la jurisprudence européenne en matière de droits politiques et de partis politiques mais, en jugeant le présent à l'aune du passé, la Cour valide le gel de certains régimes politiques européens.

Sans reprendre les débats sans fin sur l'opportunité de la marge d'appréciation, tour à tour présentée comme outil du réalisme, de la flexibilité, du pluralisme, de l'unité dans la diversité, on peut toutefois s'interroger sur la pertinence de l'histoire comme critère de sa variabilité ${ }^{34}$. Le système conventionnel a précisément été conçu pour dépasser l'histoire, celle des régimes antidémocratiques et non libéraux qui ont fait basculer l'Europe dans la Seconde Guerre mondiale. La prochaine insertion de la marge d'appréciation au côté de la subsidiarité dans le préambule de la Convention avec la ratification attendue du Protocole additionnel $\mathrm{n}^{\circ} 15$ valide définitivement l'outil mais elle n'empêche pas de s'interroger sur la pertinence de ses critères de variabilité, dont l'histoire ${ }^{35}$.

Certes, statistiquement, la plupart des affaires qui font l'objet d'un examen au fond sur une allégation de violation de l'article 11 se soldent par une condamnation de l'État défendeur. La Cour reste très vigilante à la liberté d'association des partis politiques. Toutefois, lorsqu'elle opte pour la solution inverse, c'est souvent l'histoire qui joue le rôle décisif et provoque l'élargissement de la marge nationale d'appréciation nécessaire au renversement de la solution en défaveur du parti requérant. Dans l'arrêt Refah Partisi c. Turquie, la Cour observe que

[...] le régime théocratique islamique a déjà été imposé dans l'histoire du droit ottoman. La Turquie, lors de la liquidation de l'ancien régime théocratique et lors de la fondation du régime républicain, a opté pour une vision de la laïcité confinant l'Islam et les autres religions à la sphère de la pratique religieuse privée ${ }^{36}$.

26. Cour EDH, 30 janvier 1998, Parti communiste unifié de Turquie..., $\$ 46$.

27. Cour EDH, 13 février 2003, Refah Partisi..., $\$ 100$.

28. Cour EDH, 8 décembre 1999, Parti de la liberté et de la démocratie..., $\$ 45$

29. L. Burgorgue-Larsen, "L'autonomie constitutionnelle aux prises avec la Convention européenne des droits de l'homme», Revue belge de droit constitutionnel, $\mathrm{n}^{\circ}$ 1, 2001, p. 31-64; voir aussi D. Szymczak, «L'identité constitutionnelle dans la jurisprudence conventionnelle», in L'identité constitutionnelle saisie par les juges en Europe, L. Burgorgue-Larsen (dir.), Paris, Pédone, 2011, p. 45-60.

30. Voir J.-F. Flauss, «L'histoire dans la jurisprudence de la Cour européenne des droits de l'homme», p. 7: «Les considérations d'ordre historique particulières, de tendance historique, d'expérience historique habituellement invoquées par l'État défendeur aux fins de justifier la conventionnalité d'une ingérence dans l'exercice d'un droit garanti par la Convention sont prises en compte par la Cour européenne au titre de son contrôle sur la marge d'appréciation de l'État. Elles constituent un paramètre, parmi d'autres, de détermination de l'ampleur de celle-ci».

31. Voir V. Coussirat-Coustère, "L'activité de la Cour européenne des droits de l'homme en 1998 et 1999", Annuaire français de droit international, $n^{\circ} 45,1999$, p. $752:$ : [... l'histoire nationale fait partie de cette sensibilité collective qui, ne renvoyant à aucun standard européen commun, laisse aux autorités une large marge d'appréciation».

32. G. Cohen-Jonathan, La Convention européenne des droits de l'homme, Aix-en-Provence - Paris, Presses universitaires d'Aix-Marseille - Economica, 1989, p. 22.

33. Cour EDH, 13 février 2003, Refah Partisi..., \$104.

34. Voir M. Delmas-Marty, «Pluralisme et traditions nationales - revendication des droits individuels », in Quelle Europe pour les droits de l'homme?, P. Tavernier (dir.), Bruxelles, Bruylant, 1996, p. 81-92; E. Kastanas, Unité et diversité: notions autonomes et marge d'appréciation des États dans la jurisprudence de la Cour européenne des droits de l'homme, Bruxelles, Bruylant, 1996; S. C. Greer, La marge d'appréciation: interprétation et pouvoir discrétionnaire dans le cadre de la Convention européenne des droits de l'homme, Strasbourg, Éditions du Conseil de l'Europe, 2000.

35. Voir S. Touzé, D. Szymczak, "Cour européenne des droits de l’homme et droit international général (2017) ", Annuaire français de droit international, 2017, p. 476: "Dans la “course de lenteur" que semblent se livrer depuis quelques années déjà les Protocoles $\mathrm{n}^{\circ} 15$ et $\mathrm{n}^{\circ} 16$ additionnels à la Convention européenne des droits de l'homme en vue de leur entrée en vigueur, une légère accélération peut être signalée du côté du premier cité. Au début de l'année 2017, le Protocole no 15 comptait en effet trente-trois ratifications (dont celles de la France, l'Allemagne, le Royaume-Uni ou la Turquie), auxquelles s'ajoutent onze signatures non suivies de ratification (dont celles de la Russie, l'Italie et l'Espagne). Dès lors, seuls trois États parties ne se sont toujours pas manifestés à l'égard de ce texte, en l'occurrence la Bosnie-Herzégovine, la Lettonie et Malte».

36. Cour EDH, 13 février 2003, Refah Partisi..., \$125. 
Véritable crainte ou facilité afin de faire barrage à l'islam politique, l'histoire sert d'étai à tout le raisonnement. C'est aussi le cas dans l'affaire Rekvényi c. Hongrie du 20 mai 1999. En l'espèce, la Cour se laisse convaincre par le contexte historique hongrois, notamment l'exploitation de la police par un régime totalitaire pendant des décennies. Dès lors,

[...] le désir de veiller à ce que le rôle crucial de la police dans la société ne soit pas compromis par l'érosion de la neutralité politique de ses fonctionnaires se concilie avec les principes démocratiques

et revêt une «importance historique particulière». L'arrêt souligne par ailleurs que l'expérience du totalitarisme en Hongrie dépendait «dans une large mesure de l'engagement direct de la police aux côtés du parti au pouvoir $»^{37}$.

La Cour est encore beaucoup plus généreuse avec l'argument tiré de l'histoire et des traditions politiques ou institutionnelles lorsqu'elle statue sous l'angle de la clause politique conventionnelle, l'article 3 du Protocole $\mathrm{n}^{\circ} 1$. Il ne s'agit pas à proprement parler de la liberté des partis politiques, entendue comme liberté de créer des partis et d'y adhérer couverte par l'article 11 de la Convention, mais les ingérences dans le droit à des élections libres - liberté de voter, de candidater et protection des mandats électoraux - concernent la liberté des partis politiques. Or, l'arrêt Mathieu-Mohin et Clerfayt c. Belgique du 2 mars 1987 qui inaugure cette partie du contentieux reconnaît d'emblée aux États contractants « une large marge d'appréciation eu égard à la diversité dans l'espace, et à la variabilité dans le temps, de leurs lois en la matière $»^{38}$. Ces derniers jouissent d'une grande latitude afin d'établir, dans leur ordre constitutionnel, des règles relatives au statut des parlementaires et aux critères d'inéligibilité. Les arrêts ultérieurs stabiliseront le paragraphe suivant:

Il existe de nombreuses manières d'organiser et de faire fonctionner les systèmes électoraux et une multitude de différences au sein de l'Europe notamment dans l'évolution historique, la diversité culturelle et la pensée politique ${ }^{39}[\ldots]$,

différences «qu'il incombe à chaque État contractant d'incorporer dans sa propre vision de la démocratie ${ }^{40}$. S'agissant spécifiquement des critères d'inéligibilité, la Cour estime qu'ils sont susceptibles de varier
[...] en fonction des facteurs historiques et politiques propres à chaque État; la multitude de situations prévues dans les constitutions et les législations électorales de nombreux États membres du Conseil de l'Europe démontre la diversité des choix possibles en la matière ${ }^{41}$.

La loi électorale doit s'apprécier «à la lumière de l'évolution politique du pays, de sorte que des détails inacceptables dans le cadre d'un système déterminé peuvent se justifier dans celui d'un autre ${ }^{42}$. Aucun critère ne doit être considéré comme plus valable qu'un autre à condition qu'il garantisse, selon la lettre de l'article 3 du Protocole $\mathrm{n}^{\circ} 1$, «l'expression de la volonté du peuple à travers des élections libres, honnêtes et périodiques».

Le contexte historique a ainsi été déterminant dans de nombreuses affaires. Quelques arrêts confinent même à la complaisance et fragilisent la crédibilité des standards européens à l'instar de l'arrêt Ahmed c. Royaume-Uni du 2 septembre 1998 ou de l'arrêt $P y c$. France du 11 janvier 2005 respectivement relatifs à la participation des hauts fonctionnaires de l'administration locale à certaines formes d'activités politiques et à la condition de résidence pour pouvoir exercer son droit de vote. Dans ce dernier, la Cour tolère, compte tenu de «l'histoire et [du] statut de la Nouvelle-Calédonie» la soumission de la liberté du vote à une durée de résidence exorbitante de dix ans de résidence ${ }^{43}$.

\section{B. La fragmentation des standards démocratiques}

Outre la rigidification dommageable des régimes politiques européens, l'utilisation de l'histoire et de la tradition dans le contentieux des partis politiques possède une autre répercussion perverse. Elle favorise non pas le dédoublement mais l'éclatement de l'ordre politique européen dont les partis politiques sont un des principaux animateurs. Même si on n'adhère pas à la proposition d'un ordre juridique supra-étatique à vocation constitutionnelle qui fixerait les conditions d'exercice de la souveraineté nationale et les standards du régime politique dans les États membres, force est de constater la fragmentation des standards européens dans un domaine à l'origine et au cour des préoccupations du Conseil de l'Europe ${ }^{44}$.

37. Cour EDH, 20 mai 1999, Rekvényi c. Hongrie, $\$ 41$.

38. Cour EDH, 2 mars 1987, Mathieu-Mohin et Clerfayt c. Belgique, $\mathrm{n}^{\circ} 9267 / 81, \S 54$

39. Cour EDH, 15 juin 2006, Lykourezos c. Grèce, $\mathrm{n}^{\circ}$ 33554/o3, $\$ 51$.

40. Cour EDH, 16 mars 2006, Ždanoka c. Lettonie, $\$ 103$.

41. Cour EDH, $1^{\text {er }}$ juillet 1997, Gitonas c. Grèce, $\mathrm{n}^{\circ}$ 18747/91, 19376/92, 19379/92, 28208/95, 27755/95, \$39.

42. Cour EDH, 8 juillet 2008, Parti travailliste géorgien c. Géorgie, $\mathrm{n}^{\circ} 9103 / 04, \$ 89$.

43. Cour EDH, 11 janvier 2005, Py c. France, $\mathrm{n}^{\circ}$ 66289/01, $\$ 64$

44. Pour une opinion contraire, voir la préface de S. Touzé, in Les libertés politiques: socle pour un ordre public européen?, C. Lageot (dir.), Poitiers - Paris, Presses universitaires de Poitiers - LGDJ, 2015, p. 7: «L'ordre politique européen dans son acception la plus entière, n'existe pas et ne pourra exister tant que l'énoncé conventionnel sera limité comme il l'est actuellement et surtout, tant que les États resteront des États avec cette qualité qui les caractérise, à savoir leur souveraineté. La jurisprudence récente le met d'ailleurs en évidence avec cette imprudence teintée d'une témérité que l'on pourrait assimiler à une forme d'insouciance juridique. Cette même insouciance semble être partagée par Yannick Lécuyer lorsqu'il affirme que l'ordre politique européen repose essentiellement sur des valeurs intégratives qui pallient la carence de légitimité démocratique inhérente à la logique du droit international». On ne peut que regretter l'archaïsme de cette opinion qui réduirait presque le droit international à du droit des relations internationales, règle d'acquisition territoriale sur les îles émergentes, immunités, etc. Sous le couvert d'une notion aussi originale que mystérieuse, «l'insouciance juridique», elle fait en réalité, à la manière du siècle dernier, abstraction de toute la singularité du droit international des droits de l'homme et de l'évolution du mécanisme européen. 
Le maintien d'un « régime véritablement démocratique» ou de la «démocratie véritable» figurent tout à la fois dans le préambule de la Convention de 1950 et du Statut du Conseil de l'Europe signé à Londres le 5 mai 1949. L'ordre public européen est inévitablement impacté puisque, selon les termes employés par la Cour: «La démocratie représente sans nul doute un élément fondamental de "l'ordre public européen" » 4 .

On peut facilement délimiter les différents espaces issus de cette fragmentation. C'est d'autant plus aisé qu'ils correspondent aux grands clivages historiques et politiques du XX ${ }^{\mathrm{e}}$ siècle.

Le premier territoire correspond au «club des démocraties ", c'est-à-dire les États à l'origine du Conseil de l'Europe et de la Convention complétés par les signataires du bloc occidental pendant la guerre froide. L'histoire ne joue pas de rôle particulier. Le contrôle des ingérences dans la liberté des partis politiques conserve toute sa rigueur affichée.

Le deuxième territoire regroupe les pays d'Europe centrale et orientale. Ici, la Cour n'hésite pas à tenir compte de leur histoire spécifique afin de justifier des ingérences plus larges dans l'exercice des droits politiques et / ou de nature politique. Par histoire spécifique, il faut entendre l'adhésion au bloc soviétique pendant la guerre froide. De fait, la fracture Est/Ouest et la guerre froide se sont invitées dans la jurisprudence européenne et continuent de produire des effets bien après l'effondrement du rideau de fer. Le nivellement par le bas des standards européens redoutés par certains auteurs suite à l'adhésion de la Russie et de ces pays ne s'est pas produit ${ }^{46}$. La difficulté de certains de ces pays à adopter et s'approprier ces standards a été compensée par un morcellement jurisprudentiel au nom de la prise en compte de l'histoire. Bien qu'ancien, l'arrêt Rekvényi c. Hongrie résonne encore plus mal depuis l'arrêt Matelly c. France du 2 janvier 2015 qui sanctionne les restrictions privant les militaires, un gendarme en l'occurrence, de la liberté d'association pour la défense de leurs intérêts professionnels. Certes, il ne s'agit pas de liberté de fonder un parti politique ou d'y adhérer mais de liberté syndicale. Néanmoins les associations professionnelles sont aussi des structures basiques de la vie politique et sociale des démocraties modernes. À titre de comparaison, la Cour ne discute à aucun moment l'histoire de France ou le besoin impérieux de protéger les forces de l'ordre des influences politiques eu égard à leur collaboration à un quelconque régime antidémocratique passé. La lecture des arrêts pertinents donne le sentiment d'une jurisprudence marginale qui se nourrit d'elle-même.
Plus récemment, dans la décision Artyomov c. Russie du 7 décembre 2006, la Cour renvoie à l'arrêt Gorzelik c. Pologne pour rappeler que, eu égard au rôle particulier des partis politiques - sous-entendu la participation à la souveraineté nationale -, «les États disposent d'une grande latitude pour établir les critères de participation aux élections, lesquels varient en fonction des facteurs historiques et politiques propres à chaque État " ${ }^{47}$. L'arrêt Ždanoka c. Lettonie du 16 mars 2016 mobilise quant à lui l'arrêt Rekvényi c. Hongrie mais suggère que le morcellement des standards démocratiques serait momentané. En effet, la grande chambre estime que le « caractère totalitaire et antidémocratique des partis communistes dirigeants des États de l'Europe orientale et centrale avant 1990 " justifie une restriction "pendant les premières années suivant le rétablissement de l'indépendance de la Lettonie ${ }^{48}$. L'encadrement de cette phase transitoire avait précisément suscité la dissidence du juge Giovanni Bonello sous l'arrêt rendu en chambre, très favorable à un élargissement de la marge d'appréciation. Selon lui, il n'appartenait pas au juge de Strasbourg de se prononcer sur une question "aussi subjective et difficile à cerner que celle de savoir si la période de transition vers une nouvelle démocratie était ou non terminée en $1998{ }^{49}$. Dans cette logique, c'est aux États concernés qu'il revient de déterminer si la mesure doit être reportée ou non, ce qui leur donnerait un blanc-seing pour limiter sine die les droits politiques et démocratiques au nom du passé soviétique. L'arrêt de la grande chambre n'a probablement pas trouvé lui non plus grâce aux yeux du juge maltais. Même si, à l'inverse de l'arrêt du 17 juin 2004, elle conclut à l'absence de violation de l'article 3 du Protocole $n^{\circ} 1$, elle impose au Parlement letton de limiter la restriction litigieuse dans le temps et d'en assurer un suivi constant. Véritable "avertissement", le terme est expressément utilisé, avertissement donné «à la lumière de la stabilité renforcée dont jouit à présent la Lettonie, du fait notamment de son intégration pleine et entière dans l'ensemble européen ", toute inaction du corps législatif letton pourrait amener la Cour à revenir sur sa conclusion ${ }^{50}$.

Le troisième espace est matérialisé par la Turquie à elle seule. La singularité de la jurisprudence relative aux partis politiques turcs ne doit pas tant à la marge d'appréciation élargie en considération du contexte historique dans l'arrêt Refah Partisi c. Turquie qu'à la tolérance accrue dont bénéficient les partis favorables au séparatisme kurde. Toutes les affaires ou presque aboutissent à un constat de violation de l'article 11 sous réserve que le changement constitutionnel proposé soit envisagé de manière pacifique ${ }^{51}$.

45. Cour EDH, 30 janvier 1998, Parti communiste unifié de Turquie..., $\$ 45$.

46. S. Perez, La semaine juridique, édition générale, $\mathrm{n}^{\circ}$ 24-25, 2000-I-203, sommaires commentés, p. 273.

47. Cour EDH, 7 décembre 2006, Artyomov c. Russie (déc.), nº 14146/o2.

48. Cour EDH, 16 mars 2006, Ždanoka c. Lettonie, $\$ 74$.

49. Cour EDH, 17 juin 2004, Ždanoka c. Lettonie, opinion dissidente du juge Bonello, $\$ 3.3$.

50. Cour EDH, 16 mars 2006, Ždanoka c. Lettonie, $\$ 135$.

51. Cour EDH, 30 janvier 1998, Parti communiste unifié de Turquie...; Cour EDH, 25 mai 1998, Parti socialiste et autres c. Turquie; Cour EDH, 8 décembre 1999, Parti de la liberté et de la démocratie...; Cour EDH, 9 avril 2002, Yazar, Karataş, Aksoy...; Cour EDH, 12 juillet 2005 , Güneri c. Turquie; Cour EDH, 14 décembre 2010, HADEP et Demir c. Turquie; Cour EDH, 12 janvier 2016, Parti pour une société démocratique... 
La marge d'appréciation se réduit à nouveau à sa plus simple expression.

Enfin, le quatrième et dernier espace se situe en dehors de l'Europe géographique, dans les territoires dont les États assurent, au sens de l'article 56 de la Convention, les relations internationales, c'est-à-dire les territoires non métropolitains. Le paragraphe 3 de cette «clause coloniale» prévoit que les États peuvent étendre l'application de la Convention par déclaration à ces territoires mais que celleci sera alors appliquée «en tenant compte des nécessités locales ${ }^{52}$. Or, la Cour considère depuis l'arrêt $P y c$. France que l'histoire figure parmi les éléments principaux qui caractérisent des «nécessités locales» de nature à permettre les restrictions apportées au droit de vote ${ }^{53}$. Dès lors, les exigences conventionnelles peuvent être aménagées sans commune mesure même en matière de droits politiques et faire passer des considérations d'opportunité politique avant toute rigueur juridique ${ }^{54}$.

\section{Une vision dynamique et volontariste de l'histoire}

L'histoire des États a beau être le «ventre mou de la marge d'appréciation " 55 , la Cour est capable le cas échéant de la dépasser (A) afin de construire un droit politique commun, une histoire démocratique européenne dans laquelle les partis politiques tiennent le rôle clé et dont les libertés politiques sont le pivot (B).

\section{A. Le caractère facultatif de l'histoire des États dans le contentieux des partis politiques}

L'interprétation historique n'est pas un passage obligé. La Cour a montré sa capacité à résister à la prétention des États à «exciper d'une sorte d'exception historique [...] qui en soi légitimerait de plano une entorse au droit de la Convention $»^{56}$. L'histoire s'efface notamment lorsque la substance du droit est en jeu. Elle ne suffit pas toujours à justifier l'amplification de la marge d'appréciation et à faire basculer la décision en faveur des gouvernements défendeurs.
Premièrement, le contexte historique est parfois atténué, voire neutralisé, ce que Jean-François Flauss appelait le "gommage du poids de l'histoire ${ }^{57}$. En effet, l'auteur décelait une évolution jurisprudentielle sur ce point. Même si l'histoire continue à jouer un rôle non négligeable dans de nombreux arrêts relatifs aux partis politiques, l'argument n'a pas prospéré dans l'arrêt Vogt c. Allemagne du 26 septembre 1995. Bien qu'elle juge pertinentes les raisons avancées par le gouvernement pour justifier l'ingérence, elle estime néanmoins qu'elles ne suffisent pas à établir la nécessité de la mesure dans une société démocratique, à savoir la révocation de la requérante de la fonction publique du fait de ses engagements politiques ${ }^{58}$. Passé aux forceps, à une voix près, l'arrêt est assorti d'une série d'opinions dissidentes qui focalisent sur l'histoire et regrettent qu'elle n'ait pas davantage pesé dans la solution retenue ${ }^{59}$.

L'histoire est également neutralisée dans un certain nombre d'arrêts concernant des pays d'Europe centrale et orientale. Dans l'arrêt Partidul Comunistilor (Nepeceristi) et Ungureanu c. Roumanie du 3 février 2005, alors que le gouvernement défendeur attirait l'attention sur la «spécificité historique de la Roumanie et [...] la souffrance causée au pays par le totalitarisme communiste», la Cour souligne que ce contexte ne justifie pas à lui seul la nécessité de l'ingérence, «d'autant plus que des partis communistes ayant une idéologie marxiste existent dans plusieurs pays signataires de la Convention ${ }^{60}$. Au final, elle retient la violation de l'article 11 de la Convention car, bien que le parti requérant ait été fondé sur une doctrine communiste, ses statuts et son programme insistaient sur le respect de la souveraineté nationale, de l'intégrité territoriale, de l'ordre juridique et constitutionnel et des principes démocratiques, parmi lesquels le pluralisme politique, le suffrage universel et la libre participation à la vie politique. De plus, il ne prônait ni le soulèvement ni la violence, limites infranchissables de la tolérance dans la jurisprudence européenne. Même registre dans l'arrêt Ouranio Toxo c. Grèce, où les juges strasbourgeois concèdent que le nom du parti litigieux possède une «connotation historique négative» car il évoque la guerre civile de 1946-1949, mais concluent néanmoins à la violation de l'article 11 de la Convention ${ }^{61}$. Dans l'arrêt Linkov c. République tchèque, la Cour indique que, si le contexte historique est important, il ne lui appartient pas de se prononcer pour autant sur les faits qui se sont produits

52. Voir F. Sudre, "Le pluralisme saisi par le juge européen», in Droit et pluralisme, L. Fontaine (dir.), Bruxelles, Bruylant (Droit et justice; 76), 2007, p. 270-271; K. Grabarczyk, «Le pluralisme en trompe l'œil: la clause d'application territoriale de la Convention", in Pluralisme et juges européens des droits de l'homme, M. Levinet (dir.), Bruxelles, Bruylant, 2010, p. 151-168.

53. Cour EDH, 11 janvier 2005, Py c. France, $\$ 64$

54. Voir F. Sudre, «Droit de la Convention européenne des droits de l'homme », La semaine juridique, édition générale, n 30,27 juillet 2005 , doctr. 159.

55. Y. Lécuyer, Les droits politiques dans la jurisprudence de la Cour européenne des droits de l'homme, Paris, Dalloz, 2009, p. 494.

56. J.-F. Flauss, «L'histoire dans la jurisprudence de la Cour européenne des droits de l'homme», p. 7.

57. Ibid., p. 13 .

58. Cour EDH, 26 septembre 1995, Vogt c. Allemagne, $\$ 61$

59. Ibid., opinion dissidente commune des juges Bernhardt, Gölcüklü, Matscher, Loizou, Misfud, Bonnici, Gotchev, Jungwiert et Kuris : «En pareille situation et eu égard à l'histoire singulière de l'Allemagne, en particulier l'anéantissement de la Constitution démocratique de Weimar, l'État doit pouvoir révoquer ses fonctionnaires, y compris les enseignants, qui sont engagés activement au sein de partis antidémocratiques"; opinion dissidente du juge Jambrek: «Le système, tel qu'il découle du grand principe constitutionnel et qu'il se trouve défini par la Cour constitutionnelle allemande, repose sur une doctrine juridique étoffée et puise ses racines dans l'histoire politique allemande».

60. Cour EDH, 3 février 2005, Partidul Comunistilor (Nepeceristi) et Ungureanu c. Roumanie, n 46626/99, $\$ 41$ et 58.

61. Cour EDH, 20 octobre 2005, Ouranio Toxo et autres c. Grèce, $\mathrm{n}^{\circ} 748989 / 01, \S 39$. 
en Europe pendant la Seconde Guerre mondiale ou survenus en Tchécoslovaquie entre 1948 et $1989^{62}$. Elle concentre plutôt son contrôle sur la compatibilité des changements proposés et des moyens envisagés pour y parvenir avec la Convention et les principes démocratiques fondamentaux $^{63}$. Ce recul possible de l'histoire comme levier de variation de la marge d'appréciation a été confirmé dans le contentieux connexe du droit à des élections libres avec l'arrêt Sejdic et Finci c. Bosnie-Herzégovine du 22 décembre 2009. À l'opposé de l'arrêt Ždanoka c. Lettonie, la Cour n'accepte plus le moyen tiré de la transition démocratique pour justifier l'ingérence. L'État défendeur est mis face à ses obligations internationales:

[...] en devenant membre du Conseil de l'Europe en 2002 et en ratifiant la Convention et ses Protocoles sans réserves, l'État défendeur a librement accepté de respecter les standards pertinents ${ }^{64}$.

On peut également citer l'arrêt Adamsons c. Lettonie. Tout en gardant à l'esprit le contexte sociohistorique particulier, l'annexion du pays par l'Union soviétique et le régime totalitaire communiste, la Cour juge que, au fil du temps, ce contexte et la simple suspicion générale à l'égard d'un groupe de personnes ne suffisent plus à légitimer les ingérences aux droits politiques et de nature politique $^{65}$. Il est vrai que, contrairement à $\mathrm{M}^{\mathrm{me}}$ Ždanoka, l'appartenance du requérant au régime antidémocratique avait été jugée purement «formelle» et non «active». Bref, conformément à l'assertion de l'arrêt Hirst c. RoyaumeUni $\left(n^{\circ} 2\right)$ du 6 octobre 2005, la marge d'appréciation ne semble plus suffisante pour justifier des restrictions découlant «essentiellement d'une adhésion inconditionnelle et passive à une tradition historique ${ }^{66}$. La Cour peine à convaincre mais le principe est énoncé.

Deuxièmement, l'histoire disparaît quelquefois tout simplement de la motivation des arrêts. Elle ne constitue même plus un critère d'évaluation de l'amplitude de la marge d'appréciation. Le contexte historique n'est ainsi quasiment jamais abordé dans les arrêts relatifs aux partis politiques militant en faveur de la séparation entre la
Turquie et le Kurdistan. L'histoire de cette région est pourtant particulièrement mouvementée. Dans l'arrêt Yazar, Karataş, Aksoy et le Parti du travail du peuple (HEP) c. Turquie, l'argument est totalement absent de la motivation qui amène le juge européen à constater la violation de l'article 11 de la Convention alors qu'elle est omniprésente dans l'arrêt de la Cour constitutionnelle d'Ankara en date du 14 juillet 1993 qui dissout le parti requérant. L'arrêt du juge constitutionnel turc est annexé à celui de la Cour, ce qui est assez rare pour être mentionné ${ }^{67}$.

Troisièmement, l'histoire se retourne parfois même contre l'État qui l'invoque. Dans l'arrêt Parti pour une société démocratique (DTP) c. Turquie, le gouvernement s'est vu reprocher la dissociation des discours des dirigeants dudit parti de leur contexte historico-politique ${ }^{68}$. C'est ce qu'illustre aussi l'arrêt Partidul Comunistilor (Nepeceristi) et Ungureanu c. Roumanie. La Cour conjugue le principe dégagé dans l'arrêt Hirst c. Royaume-Uni ( $\left.n^{\circ} 2\right)$ au contexte roumain: l'expérience du communisme totalitaire en Roumanie ne saurait justifier à elle seule la nécessité de l'ingérence, «d'autant plus que des partis communistes ayant une idéologie marxiste existent dans plusieurs pays signataires de la Convention ${ }^{69}$. Elle constate par ailleurs que les statuts et le programme du parti requérant critiquaient les abus de l'ancien parti communiste avant 1989 avec lequel ils prenaient leurs distances. Autre exemple, dans l'arrêt Parti travailliste géorgien c. Géorgie du 8 juillet 2008, la Cour reproche au gouvernement de ne pas avoir étayé ses allégations historiques par des éléments concrets (rapports officiels, documents vidéo, publications dans la presse, etc.). Dans ces conditions, elle estime ne pas être capable de déterminer le poids à attacher aux événements historiques pertinents ${ }^{70}$.

\section{B. La fabrique de l'histoire politique et démocratique européenne}

Malgré les incohérences jurisprudentielles provoquées par l'histoire, il se dégage du contentieux relatif aux partis

62. Cour EDH, 7 décembre 2006, Linkov c. République tchèque, $\mathrm{n}^{\circ}$ 10504/03, $\$ 37$ et 42.

63. Ibid., $\$ 43$.

64. Cour EDH, 22 décembre 2009, Sejdic et Finci c. Bosnie-Herzégovine, no 27996/06 et 34836/06, \$49.

65. Cour EDH, 24 juin 2008, Adamsons c. Lettonie, $\mathrm{n}^{\circ} 3669 / 03, \$ 123$.

66. Cour EDH, 6 octobre 2005, Hirst c. Royaume-Uni ( $\left.n^{\circ} 2\right), \mathrm{n}^{\circ} 74025 / 01, \$ 41$.

67. Cour EDH, 9 avril 2002, Yazar, Karatas, Aksoy..., annexe, extraits de l'arrêt de la Cour constitutionnelle turque rendu le 14 juillet 1993 : «Les nations acquièrent leur existence au fil des évolutions et réalités historiques. La naissance, le développement et le renforcement des sentiments de culture commune, de solidarité sociale et de vie commune, se produisent au cours de l'histoire. La patrie du peuple qui, uni dans une structure nationale, a mené la Guerre de l'Indépendance est la Patrie turque, sa Nation est la Nation turque et son État, l'État turc. Le monde entier a utilisé le nom “Turquie" pour l'Anatolie, et ce dès le XI ${ }^{\mathrm{e}}$ siècle, et a appelé “Turcs" ses habitants. Ce fait ne signifie pas la non-reconnaissance des différents groupes ethniques faisant partie de l'entité nationale. L'histoire des individus qui constituent la Nation turque, vivent ensemble depuis des millénaires, sont unis et partagent la même culture, la même morale et la même religion, est commune. Toutes les générations précédentes ayant vécu dans le pays doivent être considérées avec les générations futures qui, sans nul doute, vont sauvegarder l'intégrité et l'honneur de la patrie et de la nation. L'identité commune et la culture de la Nation turque puisant leurs fondements dans les réalités historiques, elles ne peuvent être laissées sans protection comme c'est le cas pour l'identité et la culture de toute nation».

68. Cour EDH, 12 janvier 2016, Parti pour une société démocratique..., $\$ 104$.

69. Cour EDH, 3 février 2005, Partidul Comunistilor..., $\$ 58$.

70. Cour EDH, 8 juillet 2008, Parti travailliste géorgien c. Géorgie, $\$ 133$ : «[...] il est frappant qu'aucun document pénal pertinent n’ait été communiqué à la Cour à l'appui de l'allégation selon laquelle les individus armés supposés avoir attaqué le groupe électoral ont été reconnus coupables d'obstruction au processus électoral [...]. Le dossier de l'affaire ne contient aucun rapport ni aucune note du groupe électoral en question où figurerait un récit des faits censés s'être déroulés à la frontière administrative de la RAA [République autonome d'Adjarie] ». 
politiques une vision dynamique de celle-ci, une histoire qui s'écrit sous la plume du juge européen. La Cour ne subit plus l'inertie historique des États mais participe surtout à l'histoire démocratique de ces derniers. Ses arrêts dessinent un ius commune, un ordre juridique composé de standards démocratiques essentiels, autrement dit un ordre politique européen au centre duquel on trouve les partis politiques.

Ce ius commune démocratique s'adosse au "patrimoine juridique et politique commun » des États membres du Conseil de l'Europe des préambules du Statut du Conseil de l'Europe et de la Convention. La sauvegarde de ce patrimoine commun de traditions politiques, d'idéaux, de liberté et de prééminence du droit a quelque chose à voir avec la réalisation des objectifs et des idéaux du Conseil de l'Europe, c'est-à-dire l'instauration d'un «ordre public communautaire des libres démocraties d'Europe $»^{71}$. Son exploitation jurisprudentielle est aussi précoce que constituante pour le contentieux européen. Il est invoqué dès 1961 dans la décision Autriche c. Italie dans laquelle la Commission européenne des droits de l'homme l'érige en «nécessité pour les libres démocraties européennes». Il sert de pivot à l'arrêt Parti communiste unifié de Turquie c. Turquie afin d'étendre l'applicabilité de l'article 11 de la Convention aux partis politiques. La Cour se réfère au

[...] patrimoine commun d'idéal et de traditions politiques, de respect de la liberté et de prééminence du droit dont les constitutions nationales fournissent d'ailleurs souvent une première concrétisation ${ }^{72}$.

Sous-entendu, le juge européen assure le second niveau de concrétisation. On le retrouve au cœur du paragraphe le plus important, celui qui fera passer l'arrêt à la postérité parce qu'il incorpore la démocratie à l'ordre public européen:

La démocratie représente sans nul doute un élément fondamental de «l'ordre public européen» (arrêt Loizidou précité, p. $27, \$ 75$ ). Ceci ressort d'abord du préambule à la Convention, qui établit un lien très clair entre la Convention et la démocratie en déclarant que la sauvegarde et le développement des droits de l'homme et des libertés fondamentales reposent sur un régime politique véritablement démocratique d'une part, et sur une conception commune et un commun respect des droits de l'homme d'autre part (arrêt Klass et autres précité, p. 28, \$59). Le même préambule énonce ensuite que les États européens ont en commun un patrimoine d'idéal et de traditions politiques, de respect de la liberté et de prééminence du droit. La Cour a vu dans ce patrimoine commun les valeurs sous-jacentes à la Convention ${ }^{73}$.
Combiné à l'objectif «d'une union sans cesse plus étroite » entre les membres du Conseil de l'Europe, également énoncé dans le préambule de la Convention, le patrimoine sert de support à la constitution d'un espace politique et démocratique commun et confère implicitement un mandat à la Cour pour construire l'avenir démocratique de l'Europe. Dès lors, la Cour devient plus actrice que spectatrice de l'histoire comme elle l'est lorsqu'il s'agit d'encadrer la marge d'appréciation des États.

Cette vision dynamique et constructive de l'histoire recèle une peur primordiale sous-jacente: le retour des régimes antidémocratiques contre lesquels la Cour est chargée de faire barrage. C’est précisément ce qui ressort des arrêts Refah Partisi c. Turquie, Vona c. Hongrie ou des décisions Artyomov c. Russie, Staatkundig Gereformeerde Partij c. Pays-Bas du 10 juillet 2012... Dans ces arrêts, l'histoire est d'abord un outil d'identification des idéologies dangereuses que le juge européen doit aider à juguler et qui font peser un danger réel et effectif sur certaines sociétés. En effet, l'arrêt Refah Partisi c. Turquie regarde davantage vers l'avenir que le passé. La Cour constate, à l'aune de l'histoire de la Turquie mais aussi de «l'histoire européenne contemporaine», que la mise en œuvre du projet politique du parti requérant était réaliste en raison de son influence, de ses résultats électoraux, de ses chances réelles d'arriver au pouvoir ${ }^{74}$. Dans ces circonstances, il fallait éviter à tout prix les bégaiements de l'histoire, c'est-à-dire le retour aux responsabilités d'un mouvement politique théocratique et fondamentalisme. L'arrêt Vona c. Hongrie fait application des mêmes principes à propos de la Garde hongroise pour la défense des traditions et de la culture, mouvement d'extrême droite directement inspiré du parti nazi, pro-germanique et antisémite des Croix fléchées pendant la Seconde Guerre mondiale et prônant la division raciale ${ }^{75}$. L'irrecevabilité des requêtes dans les décisions Artyomov c. Russie et Staatkundig Gereformeerde Partij c. Pays-Bas n'est pas non plus étrangère à l'idéologie affichée par ces partis politiques: une affiliation pan-nationale et ethnique dans le premier cas, une conception traditionaliste, sexiste et discriminatoire des relations entre sexes dans le second. L'arrêt Ždanoka c. Lettonie peut se lire de cette manière mais avec des différences notables ${ }^{76}$. Tout d'abord, il ne s'agissait pas d'un mouvement politique antidémocratique capable de faire basculer le régime démocratique mais d'une candidate aux élections parlementaires. Le risque de restauration d'un régime totalitaire en Lettonie du seul fait de $\mathrm{M}^{\mathrm{me}}$ Ždanoka était par conséquent discutable et la transposition des

71. Commission EDH, 11 janvier 1961, Autriche c. Italie (déc.), nº 788/60.

72. Cour EDH, 30 janvier 1998, Parti communiste unifié de Turquie..., \$28.

73. Ibid., $\$ 45$.

74. Cour EDH, 13 février 2003, Refah Partisi..., $\$ 99$ et 110.

75. Cour EDH, 8 juillet 2013, Vona c. Hongrie, $\mathrm{n}^{\circ} 35943 / 10, \S 65$.

76. Cour EDH, 16 mars 2006, Ždanoka c. Lettonie, $\$ 133:$ «Si pareille restriction ne peut guère être admise dans le contexte d'un système politique donné, tel que celui par exemple d'un pays qui est doté d'un cadre établi d'institutions démocratiques depuis des dizaines d'années ou plusieurs siècles, elle peut être jugée acceptable [...] compte tenu du contexte historico-politique ayant conduit à son adoption et de la menace que représente pour le nouvel ordre démocratique la résurgence d'idées qui risqueraient de conduire à la restauration d'un régime totalitaire si on les laissait gagner du terrain ". 
principes énoncés dans l'arrêt Refah Partisi c. Turquie beaucoup moins pertinente. Le «revirement» entre la Chambre de sept juges et la grande chambre trahit surtout un manque de confiance dans la société démocratique lettone. L'arrêt de Chambre possédait deux avantages. Premièrement, il était plus cohérent avec la solution adoptée dans l'arrêt Vogt. Deuxièmement, il proposait une vision plus optimiste de l'ordre public et de l'ordre politique européens.

L'utilisation de l'histoire dans ces décisions présente un lien de parenté avec le contentieux relatif aux «vérités historiques » dès lors qu'il s'agit en réalité de tentatives de réhabilitation du régime national-socialiste, de négation de crime contre l'humanité, de diffamation raciale et d'incitation à la haine ${ }^{77}$. Même lorsqu'il n'est pas appliqué, l'ombre de l'article 17 de la Convention plane sur cette partie du contentieux très spécifique. Conçu comme une arme contre les requérants de ce type, il précise qu'aucune des dispositions de la Convention

[...] ne peut être interprétée comme impliquant pour un État, un groupement ou un individu, un droit quelconque de se livrer à une activité ou d'accomplir un acte visant à la destruction des droits ou libertés reconnus dans la présente
Convention ou à des limitations plus amples de ces droits et libertés que celles prévues à ladite Convention ${ }^{78}$.

L'arrêt Vogt c. Allemagne reconnaît d'ailleurs la légitimité des ingérences étatiques destinées à instaurer une « démocratie apte à se défendre » afin d'éviter les errances du passé 79 . C'est précisément ce que pratique la Cour dans le contentieux des partis politiques, de manière juridictionnelle et à une échelle supranationale.

En conclusion, l'emploi de l'histoire dans le contentieux des partis politiques répond à deux objectifs distincts : l'extension parfois erratique de la marge d'appréciation des États dans un contentieux où le contrôle est censé être optimal d'une part, la préservation d'une Europe démocratique d'autre part.

Dans les deux cas, l'argument historique ramène surtout aux origines du système conventionnel de protection des droits de l'homme et des libertés fondamentales. Elle le remet en perspective, convoque sa cause première et le projette vers l'avenir, ce qui n'est pas un luxe à l'heure où les mouvements nationalistes, racistes et extrémistes fleurissent à nouveau en Europe et où la Cour traverse une crise de légitimité sans précédent.

77. Cour EDH, 24 juin 2003, Garaudy c. France (déc.).

78. Voir A. Spielmann, «La Convention européenne des droits de l'homme et l'abus de droit», in Mélanges en hommage à Louis Edmond Pettiti, Bruxelles, Bruylant, 1998, p. 673-686; G. Cohen-Jonathan, "Abus de droit et libertés fondamentales », in Mélanges en l'honneur de Louis Dubouis : au carrefour des droits, Paris, Dalloz, 2002, p. 517-544; M. Levinet, «Le pluralisme confronté à la clause d'interdiction de l'abus de droit de l'article 17 de la Convention européenne des droits de l'homme ", in Pluralisme et juges européens des droits de l'homme, p. 125-150.

79. Cour EDH, 26 septembre 1995, Vogt c. Allemagne, $\$ 51$. 